\title{
Gender Reassignment Surgery
}

\section{Tamara C McGill-Carter*}

The Chicago School of Professional Psychology, US

*Corresponding Author: Tamara C McGill-Carter, The Chicago School of Professional Psychology, US.

Received: March 27, 2019; Published: July 19, 2019

DOI: $10.31080 /$ ASNE.2019.02.0084

\begin{abstract}
This paper discusses the moral, ethics, and medical permissibility of undergoing Gender Reassignment Surgery. The cons listed are in the opinions of feminists who advise against such unethical bodily damage as one should be proud to be the gender they were born with. But it is within the moral judgment of those same feminists that does advise for Gender Identity Disordered individuals to seek out psychiatric services rather than turn to a rather risky and often times, irreversible medical procedure!
\end{abstract}

Keywords: Ethics; Moral; Medical; Gender; Disorder; Identity; Psychiatric

\section{Introduction}

Traditionally, gender is biologically defined as male or female as given to the developing baby by the father's sperm, him being the determinant of the child's gender by either the XX or XY chromosomes. Once the child is born, the parents are informed of what gender their child is and are able to ascertain how to accommodate their new bundle by either dressing him or her in either pink or blue. But what would happen if this gendered individual later on in their lifespan develops a cognitive dissonance that would change how he or she feels about him/herself as a person? Or their gender?

The question of what to do when a gendered individual experiences cognitive dissonance regarding his/her assigned gender actually be answered in a number of ways with Gender Identity Disorder being the first of the many later changes an individual can take to feel more like their rightful gender inside as well as out [1]. While gender identity disorder begin with the dissonance, sex reassignment surgery is often the ultimate choice and gives the dissonanced individuals more free choices to become what they have been "feeling", but is GRS really a great idea so to achieve the rightful gender and remove the dissonance?

To those whom are looking to change genders may not honestly be considering the ethics [2], their as well as the surgeon's moral permissibility for/against performing such a risky procedure [2], the patient's possible satisfaction/dissatisfaction of the job after the procedure is complete [3], their psycho-social outcome having become the gender they wish [4,5], and finally, the psychiatric contributions to those that have undergone the surgery and are living with their new gender [6]. Evaluations are normally done with the newly sex changed individuals that assess what created the dissonance by using a gendergram to go through one's lifespan to pinpoint the exact moment the dissonance began. There are many factors involved in changing genders and will be evaluated in great detail as we continue.

\section{Cognitive dissonance}

Beginning with the cognitive dissonance of the individual whom is the gender they were born with, a Gender Identity Disorder can be classified as described in the DSM IV-TR [1] as a strong and persistent cross-gendered identification, not merely a desire for perceived cultural advantages of being the other sex.

In children, the disturbance is manifested by four or more of the following criteria (1) repeatedly stated desire to be, or insistence that he or she is the other sex, (2) in boys, preference for cross dressing or simulating female attire; in girls, insisting on wearing on stereotypical masculine clothing (3) strong and persistent preferences for cross-sex roles in make believe play or persistent thoughts about being the other sex. While many more formalities of the criteria is listed, the general rule of thumb is that the disturbance 
must have a form of impairment on one's social, cognitive, and occupational functioning in order to be considered as such a disorder. For children, the diagnostic code for Gender Identity Disorder is 302.6 and the code for teens to adults is 302.85 [1]

After identification of the full diagnosis of a GID, gender reassignment surgery is often brought to the attention of a psychiatrist and/or plastic surgeon by more men than women as the desire to be a woman is more often documented than women who desire to be men (Fitzgibbons, Sutton and Leary, 2009). However it has been documented that GRS is often the involvement of the gender identity disorder rather than treating it (Fitzgibbons., et al. 2009). Originally GRS was designed for fixing deformed or malfunctioning organs that the individual was born with, but the surgical procedure has expanded now to even destroying healthy or even malfunctioning organs in those individuals who desire GRS (Fitzgibbons., et al. 2009).

\section{The choice to reassign}

For both males and females, the procedure for GRS is both risky and often times irreversible. For example, for men to become sex changed women, a number of areas have to be touched on such as the man dressing as a woman in public, undergoing electrolysis to remove facial and genital hair, but first undergoing estrogen hormone treatment for a number of months so to disrupt the testosterone in their system to prepare the genital tissue (Penis and the testes) to be changed to a make shift vagina, complete with vaginal opening and cervix (Fitzgibbons., et al. 2009). For those men whom begin the process are often dissatisfied with the initial cosmetic surgery results because those men whom are looking to be women seek to be changed into stunning females and are often disappointed with the results of their cosmetic change that did not meet their expectations (Fitzgibbons., et al. 2009).

For females looking to have their genders changed to men involves the same procedure as men, but done in a different fashion as much more has to be done with the woman's body before she can undergo the cosmetic surgery to get her desired outcome (Fitzgibbons., et al. 2009). First, much like the man, she must undergo hormone treatment to disrupt the flow of estrogen in her body so to accommodate the incoming testosterone. After which, body changes are then made such as the breasts being removed (first by being bound), total hysterectomy, and the then space is partially closed to create a pseudo penis and testes.

It is note worthy after all that increasing the testosterone in the woman's body will cause a change in personality, including making the woman more aggressive (Fitzgibbons., et al. 2009). The hysterectomy is then performed to stop the menstruation, which for many, removes the unwanted monthly evidence for womanhood and vulnerability. Relatively few women undergo GRS, even those with severe gender dysphasia, chose to take the last step which would be the creation of a pseudo penis and testes. When this is done, the artificial organs are often small and non-functional. A penis maybe constructed to enable a mechanical erection and the simulation of sexual intercourse, but the ejaculation is not at all possible. While the surgeons attempt to preserve sexual sensations in the newly constructed organs, they are not always successful. Ultimately it is virtually impossible to "change" genders as one's gender is structured in the genetic DNA as is determined by the offspring's father at XX or XY gene combination. The change is only evident physically as one undergoes the surgery (Fitzgibbons., et al. 2009).

While one will consider GRS so to be a new gender inside and out, the morals and ethics of such a change are often overlooked by both the surgeon and the individual whom requests this change [2]. Feminists have tried to stress the importance of why such surgery isn't necessary while also providing numerous scenarios to the pros and cons of such surgery, but mostly the cons. While feminists are not totally against the changes one wishes to make to themselves to enhance their lives, they are, however, not fond of the surgeons who are more than willing to perform such a procedure without considering the morals and ethics of doing so $[2,6]$.

\section{The ethics of reassignment}

The arguments for the moral and ethical permissibly for Gender Reassignment Surgery, though some surgeons claim that such surgery has no place as a morally acceptable possibility in the treatment of GID, or alleviating the symptoms of said disorder, patients are often directed to a specialized psychiatrist first to get a "mental" check up before any procedures are followed through on $[2,6]$. The surgeons and specialized psychiatrics often look to the bioethical principals that can lead to the conclusion that it is indeed morally permissible if certain conditions are met as described by the psychiatrist. The only way GRS can be the primary, ethically, and morally acceptable way is if it has its basis in the patient autonomy, an idea that comes straight from Kantian Reasons, meaning reasonable and/or rational. So if he or she is free and clear of conscience, then it is to reason that he or she can make competent decisions about their healthcare and well being. Competent adults who have identified themselves as transsexuals have the right to self determination to make the decision on gender reassignment surgery.

Competence is always key when it comes to who should be able to obtain access to GRS [2]. The decision to undergo GRS is not one lightly or hastily made; rather it is often the final step towards 
completion of a long and hard journey of identity realization as a member of the opposite sex [2,6]. While some individuals stop at cross-dressing or even hormone therapy, others continue to request GRS. All humans go through different stages in realizing their identities, but some who are gender variant, this can be much more difficult since society is generally quite intolerant of gender categories outside of the gender binary.

Now while the Kantian Reasoning speaks for the reasons for Gender Reassignment Surgery, there are arguments against such a procedure that feminist mostly outweigh the cons of such a change more than the pros [25]. The first objection would be that it is morally wrong for a physician to remove healthy functioning organs from a patient under any circumstances on such basis that said actions are in congruous with the goals of medicine. Feminists who make this objection against GRS argue that removing healthy organs for non-prophylactic reasons is an action to ethical and moral duties of the physicians of first do no harm (Oath of Hippocrates) $[2,5]$.

It is not sound to remove unhealthy organs for not ethical or medical reason at all. Therefore, proponents of this idea argue that undergoing GRS is unsound by any means thought of and thus is immoral and should not be permitted.

Another reason for objection to the argument that GRS is morally permissible lies within the Daniel Callahan's definition of health that states health as having to do solely with physical states [2]. Currently transsexuality is classified as psychiatric disorder by those in the psychiatric and medical community, thus some may/ will object to the argument for GRS on the grounds that surgery should not be used as a cure for disorders that are psychiatric or psycho-social. To consider surgery as a possible treatment option for GID and/or GRS would be to medicalize gender variance. Therefore to have a medical solution to a non-medical problem is not only wasteful of medical resources, but also entirely illogical [2].

The final argument against the moral permissibility of GRS as a treatment option also lies within the doctor/psychiatrist/medical team that will be willing to treat the physical symptoms of a mental disorder based on what would make the patient "feel" better or whether the psychiatric symptoms that is causing the disorder should be treated only while leaving the surgery option out completely [2]. An example of this would be for a doctor to encourage a patient suffering with anorexia nervosa, also a psychiatric disorder, to continue to starve him or herself on the basis that being thinner would make that person "better". To do so would be medically negligent and a class action suit would immediately follow that doctor's negligent encouragement. Therefore it is not encouraged for one to undergo the GRS surgery no matter how tempting the offer is.

While the surgery does help one achieve the desired gender so to feel more comfortable with one's self, Fleming, McGowan, Robinson and Spitz [3] gathered twenty two participants whom have transcended from female to male transsexuals and measured each member's psychological state of mind and body satisfaction having undergone the surgery [3]. There were no significant effects on the self- esteem measure: All group means fell on the high self-esteem side of the scale. Only moderate correlations were found between positive body image and high self-esteem for control males, the no- hysterectomy group, and the hysterectomy group. On the other hand, a strong correlation with body image and self esteem was found for the phalloplasty group, indicating that those under this group were very happy with their assigned reassignment [3]. In general, a tendency was found toward increased body satisfaction with increased amount of surgical reassignment. The phalloplasty group (most reassignment) was not significantly different from the control group. The hysterectomy group showed high satisfaction on many items and their satisfaction was consistently greater than that of the no hysterectomy group. This result suggests that the hysterectomy may have a significant positive effect on the transsexual's body image even though the outward appearance of the body was not changed in any way [3]

\section{Reassignment case study}

Psychiatry and medical plastic surgery have always been very separate fields of medicine. However considering much of one's psychological outcome is important for patients whom have decided to undergo sex reassignment surgery, psychologist have been involved in the consultation of patients whom have decided to swap their gender because of the high rates of suicides that have a history of taking place if one decides to return to their original gender and later learns that the process not always reversible, especially the female to male/male back to female surgery [6].

In a case study done on patient H.T. whom underwent sex reassignment surgery due to feeling more feminine has long wanted to change his gender for the past five years past the year 1986 . He had been seen and turned down by two other gender dysphasia programs prior to coming to the center. H.T. presented at the clinic now requesting an evaluation of his appropriateness for surgery subsequent to following recommendations made 1 year ago that he should cross dress $100 \%$ of the time to become comfortable in the 
feminine role, become involved in psychotherapy with a therapist in his home community, and have electrolysis done. These recommendations are standard ones for patients seeking male to female sex reassignment surgery. Having revealed to the surgeon about his frequent sexual activity with males, but did not want to be seen as a homosexual, H.T.'s sexual identity was brought to question. In turn, the surgeon did warn H.T. that the orgasmic potential will diminish with the surgery. H.T. argued against the surgeon's warning and demanded to have the surgery anyway [6].

In H.T.'s case, a gendergram was evaluated by a psychiatrist to pin point when he became feeling "unlike a man" and found it to be within his childhood when he use to play dress up with his sister at the age of five years. Since then, H.T. felt that he was more feminine than masculine, thus resulting in a cognitive dissonance of his gender.

Clinical experience of plastic surgeons and collaborating psychologists has contributed some general guidelines about which candidates were likely to be dissatisfied with their surgeries and those candidates are those who expectations are unrealistic, whom has had previously unsuccessful procedures, or who is responding to the pressures of others to have their appearance altered [6].

\section{Post operative effects of gender reassignment surgery}

The data collected on this particular demographic of sex-reassigned and control persons prior to study entry were specifically seeking the mortality and post-op psychiatric disorders [7]. From the first set of data that was collected, there were no substantial differences between female-to-males and male- to-females mortality and psychiatric disorders of the baseline characteristics. However, immigrant status for mortality and psychiatric disorders were twice as high among transsexual individuals compared to controls [7]. Transsexual individuals were reported to be hospitalized for psychiatric morbidity and not specifically for gender identity disorder prior to sex reassignment. To adjust for these baseline discrepancies, hazard ratios were adjusted for immigrant status and psychiatric morbidity prior to measuring the baseline presented for all outcomes [7].

Individuals that underwent gender assignment surgery had a higher risk of inpatient care for a psychiatric disorder other than gender identity disorder than did the control co-horts [7]. In line with the increased mortality from suicide, gender reassigned individuals were also at a higher risk for suicide attempts, though this was not statistically significant for the time periods of 1989 throu- gh 2003 [7]. The risks of being hospitalized for substance misuse or accidents were also not significantly correlated with post-operative gender reassignment surgery.

\section{Conclusion}

In summary, while Gender Reassignment Surgery maybe some individual's only chance to become what they are hoping to be, female to male/male to female, there are many ethical, moral, and even psychological guidelines that one must consider before just "jumping to" the procedure. All rules, regulations, and considerations under the Katain Reasoning should be evaluated and if by an expert psychiatrist one is deemed unsound of mind, they will not be considered candidates for the surgery, but more to be fully psychologically evaluated for the symptoms of their Gender Identity Disorder.

\section{Bibliography}

1. American Psychological Association. Diagnostic Statistical Manual of Mental Disorders Fourth Edition Text Revision. Washington DC (2000).

2. Hume CM. "Sex, Lies, and Surgery: the Ethics of Gender Reassignment Surgery”. Res Cogitans (2011): 237-248.

3. Fleming M., et al. "The body image of post operative female to male transsexual". Journal of Consulting and Clinical Psychology 50 (1982): 461-462.

4. Abramowitz SI. "Psycho-social outcomes of Sex reassignment plastic surgery". Journal of Consulting and Clinical Psychology 54.2 (1986): 183-189.

5. Buss DB and Schmitt PD. "Evolutionary Psychology and Feminism: Sex Roles". Department of Psychology, University of Texas (2011): 1-20.

6. Deaton AV and Langman MI. "The contributions of psychologists to the treatment of plastic surgery patients". Professional Psychology: Research and Practice 17 (1986): 179-184.

7. Dhejne C., et al. "Long-term follow up of transsexual persons undergoing sex reassignment surgery: Cohort study in Sweden". PLOS ONE 6 (2011).

\section{Volume 2 Issue 8 August 2019 (C) All rights arereserved by Tamara C McGill-Carter.}

